\title{
MEASUREMENT OF THE SHORTEST PATH LENGTH; DISTANCE ESTIMATION WITHIN THE 3D BORDERS OF A TISSUE OF INTEREST
}

\author{
Bouke A De Boer ${ }^{1,2}$, Frans PJM VoOrbraAK ${ }^{2}$, Gert VAN DEN BERG ${ }^{1}$ AND JAN M \\ RUIJTER ${ }^{1}$ \\ ${ }^{1}$ Heart Failure Research Center, Department of Anatomy, Embryology and Physiology, Academic Medical \\ Center, Meibergdreef 15, 1105AZ Amsterdam, Netherlands; ${ }^{2}$ Department of Medical Informatics, Academic \\ Medical Center, Meibergdreef 15, 1105AZ Amsterdam, Netherlands \\ e-mail: j.m.ruijter@amc.uva.nl (corresponding author), b.a.deboer@amc.uva.nl,f.p.voorbraak@amc.uva.nl, \\ g.vandenberg@amc.uva.nl \\ (Accepted October 9, 2009)
}

\begin{abstract}
Volume data, such as 3D reconstructions from histological sections or MRI and CT data, are commonly used in studies in biology and medicine. The quantification of morphological parameters and changes within a region of interest is a key concern in such studies. Specifically, it is often required to measure the distance between two points. These distance measurements have to follow a track through the tissue when measuring in sheetlike or contorted organs like the developing heart. A tool was developed that enables this kind of distance measurements. Three existing neighborhood estimators were compared; two of Verwer and one of Kiryati, all originally designed to compute chamfer distances in data sets with isotropic, cubic voxels. The estimators were therefore adjusted to handle non-isotropic data sets. Moreover, the shortest path along a track within a given tissue was calculated. The measurement of known distances, through a simplified model of an early heart tube, with anisotropic voxels was used decide which of the three estimators should be implemented. The observed Root Mean Square (RMS) errors were similar to the ones reported in literature in the unrestrained isotropic case. The adjusted Verwer estimator measuring in a $5^{3}$ neighborhood performed best by far with the lowest mean and RMS errors.
\end{abstract}

Keywords: 3D reconstructions, distance estimation, non-convex, anisotropic.

\section{INTRODUCTION}

In biology and (bio)medicine volume data, such as $3 \mathrm{D}$ reconstructions from histological sections or MRI and CT data, is commonly used and the quantification of morphological parameters and changes within a region of interest is a key concern. Geometric parameters, like volume, length and number of distinct tissue components can be estimated using stereological methods (Howard and Reed, 1998), while morphogenetic parameters like cell proliferation and cell size can be quantified and 3D visualized using more recently published methods (Soufan et al., 2001; 2006; 2007).

The latter studies showed that the cells inside the early developing heart do not proliferate while the heart itself is growing rapidly. Therefore, a key question in the study of the embryonic heart is how this primitive heart grows. We hypothesized that the addition of cells from surrounding tissue was responsible for this rapid growth. To test this hypothesis it was needed to measure the speed by which cells migrate into the heart and thus to compute the distance traveled by these cells during a specified period of time (van den Berg et al., 2009).
Such migration of cells between and within organs and tissues is one of the processes in control of morphogenesis. The measurement of the length of migration paths through the tissue is required for the quantification of this process. These distance measurements have to follow a track through the tissue in a non-convex space. This is especially true when distances are measured in sheet-like or contorted organs, like the developing heart.

The software currently used for making reconstructions, such as Amira, only allows the measurement of Euclidean distances in the convex space. However, measurement of distances in the non-convex space, thus taking tissue boundaries into account, is not possible in any of the available software packages. To fill this gap we decided to develop a tool that enables such measurements.

Existing distance estimators form the basis of our tool. In the past, these algorithms for distance estimation were thoroughly investigated. Most published estimators are restricted to 2 dimensional images and are used to compute distance transformations. Distance computations in volume data with the purpose to compute 
a shortest path are only dealt with in a few articles (Kiryati and Szekely, 1993). The 2D and 3D algorithms are mostly designed to handle data sets with isotropic, cubic, voxels. Handling of nonisotropic datasets is described by Coquin and Bolon (1995) for pixels and Sintorn and Borgefors (2004) and Fouard and Malandain (2005) for voxels.

Chamfer distances are commonly used to estimate distances (Borgefors, 1984). Chamfer distances are based on the principle that the distance from one pixel or voxel to another can be determined via its neighbors. The values assigned to each neighbor relation should be carefully chosen to reach an unbiased estimation and small estimation errors. Chamfer distances have been described for the computation of distance transform images and for estimation of a shortest path. The difference between these approaches is the underlying algorithm. For distance transformation the distance is estimated as the distance from each position within an image to the nearest object within it. This estimation can be performed relatively fast by propagating forward and backward through the image once (Borgefors, 1984). In contrast to distance transforms, shortest paths do not follow straight lines but are restricted by object boundaries. When estimating such shortest paths, an algorithm should be used which propagates through the image from a starting point. Such an algorithm can be based on Dijkstra's shortest path algorithm (Dijkstra, 1959).

Verwer (1991) found optimal values to compute chamfer distance transformations by minimizing the maximum error as well as the unbiased mean square error. These optimal values were determined using a set of Euclidean distances from the origin to an Euclidean circle or ball, for two and three dimensions, respectively. Kiryati and Szekely (1993) optimized the values for estimation of the shortest path using a set of digitized surfaces. The distance estimation values found by Kiryati and Verwer are given in Fig. 2 .

The paths and distances we want to measure in the developing heart are neither straight lines, nor are they digitized surfaces. Knowing that the early primitive heart tube does look like a kind of folded sheet we expected that the approach of Kiryati and Szekely might give the best results. They, however, determined only a set of estimator values for the $3^{3}$ neighborhood, while Verwer also found a solution for the $5^{3}$ neighborhood which had a significantly lower Root Mean Squared (RMS) error (Verwer, 1991).

The performance of the different estimators, which we adjusted for anisotropy, was tested. Using test models having different degrees of anisotropy, we based our tests on the RMS errors and mean errors (bias) of the estimators. In all tests the adjusted $5^{3}$ neighborhood estimator of Verwer performed best. The distances required to answer the biological question of van den Berg et al. (2009) were measured using this estimator.

\section{MATERIAL AND METHODS}

\section{TEST MODEL}

We designed a model with the geometric characteristics of a primitive heart tube to compare the performance of the different estimators. Similar to a real primitive heart tube (Fig. 9A), our model (Fig. 1) had a height of $600 \mu \mathrm{m}$, an outer diameter of $309 \mu \mathrm{m}$ and a wall thickness of $18 \mu \mathrm{m}$. We "unfolded" the test model to compute the expected distances; transforming the tube to a plane with a width equal to the inner circumference of the tube (Fig. 1) and a height equal to the height of the tube. The expected distances for lines making an angle of $0^{\circ}$ to $90^{\circ}$ with the $x y$-axis were then easily computed. Relative to these expected distances the performance of the different adjusted estimators was tested in terms of Root Mean Squared (RMS) errors and bias (mean errors)

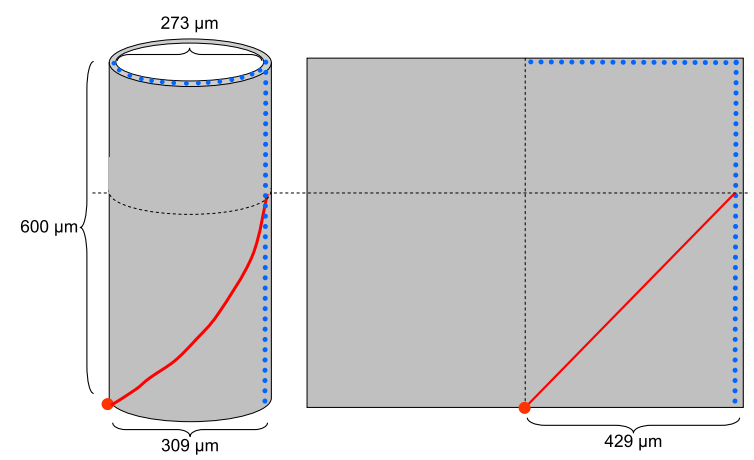

Fig. 1. The model, representing the embryonic heart tube, used to test the performance of the adjusted distance estimators trough non-convex space with anisotropic voxels. Its unfolded counterpart is shown to illustrate the calculation of the expected distances.

\section{ANISOTROPY EFFECT}

The effect of different degrees of anisotropy was determined using a constant voxel size in the $x y$ direction of $6 \mu \mathrm{m}$ and three different voxel sizes $(6$, 7.5 , and $12 \mu \mathrm{m}$ ) in the $z$ direction.

To test whether it is better to down-sample volume data when having a voxel size that is much smaller in the $x y$ direction than in the $z$ direction we also tested voxel sizes of $3 \mu \mathrm{m}$ in the $x$ and $y$ direction and $6 \mu \mathrm{m}$ 
in the $z$ direction and compared the results with the test with the cubic voxels of $6 \times 6 \times 6 \mu \mathrm{m}^{3}$.

Down-sampling to reach isotropic voxels is sometimes hampered by tissue components that are too thin to avoid their partial loss. The use of models with anisotropic voxels is then unavoidable. Our microscopic images have a resolution of $1.22 \times 1.22 \times$ $7 \mu \mathrm{m}^{3}$. To compare this degree of anisotropy with the other tests, voxel sizes were used of $3 \mu \mathrm{m}$ in the $x$ and $y$ direction and $20 \mu \mathrm{m}$ in the $z$ direction.

\section{DISTANCE ESTIMATORS}

We tested the performance of the $3^{3}$ neighborhood estimator of Kiryati and Szekely (1993), called Kiryat $_{3}$ estimator in this text, and the $3^{3}$ and $5^{3}$ neighborhood estimators of Verwer (1991), dubbed Verwer $_{3}$ and Verwer $_{5}$ estimator, respectively. Euclidean chamfer distances in the $3^{3}$ neighborhood, Euclidean $_{3}$, are included for illustrating purposes. All estimators were adjusted for anisotropy as shown in Fig. 2.

\section{IMPLEMENTATION}

Matlab (The Mathworks, Inc.) was used to implement most functions, tests, and a user interface. Only a priority queue was implemented in Java to enable propagation through the voxels within the tissue while assigning distance values. To compare the adjusted distance estimators, each estimator was implemented using Dijkstra's shortest path algorithm (Dijkstra, 1959) to determine the shortest path based on the values of the studied estimator. Note that the different estimators will not result by definition in the same shortest path. The algorithm used was implemented as follows:

for all position neighboring startposition do compute distance from startposition enqueue position

\section{end for}

currentpos $\leftarrow$ queиe.dequeue

while current pos $\neq$ end position $\& \&$ queue $\neq \varnothing$ do for all position neighboring current pos do compute distance from current pos

if distance to position was computed \&\& distance to position in queue $>$ distance to position then

delete position from queue

\section{end if}

enqueue position

\section{end for}

currentpos $\leftarrow$ quеие.dequeue

end while

trace back path from endposition to startposition

\section{BIOLOGICAL APPLICATION}

It was recently shown that the early heart tube does not proliferate (Soufan et al., 2006) and that growth of this tube is therefore due to addition of cells from the dorsal mesoderm (van den Berg et al., 2009). Because the newly added cells have recently divided they can be labeled with BrdU which is incorporated into nuclei prior to division. To this end, chicken embryos were exposed to BrdU in ovo. After predetermined exposure times, these embryos were harvested, fixed and embedded in paraplast. Serial sections were stained to enable discrimination between myocardium, BrdU-labeled nuclei, and all nuclei (Soufan et al., 2007; van den Berg et al., 2009).

The resulting sections were used to generate quantitative 3D reconstructions of the BrdU positive nuclear fraction (Soufan et al., 2007). In this timed series of reconstructions (Fig. 9), a widening zone of high BrdU-positivity visualizes the migration of newly divided cells into the heart (van den Berg et al., 2009). The voxels of the reconstructions were down-sampled to $6.1 \mu \mathrm{m}$ in the $x y$ plane and $7 \mu \mathrm{m}$ in the $\mathrm{z}$ direction to measure the lengths of the paths, from the base to the top of the migration front.

\section{RESULTS}

\section{ADJUSTMENT OF THE ESTIMATOR VALUES FOR ANISOTROPY}

The anisotropic voxel sizes made it necessary to adjust the estimator values used to compute the local distances. The optimized local distances in the published estimators $\left(D_{\text {opt }}\right)$ which are based on voxels of length 1 in the $x, y$ and $z$ direction form the basis for this compensation (Fig. 2). The actual Euclidean distances are computed for the anisotropic voxels in the $3^{3}$ or $5^{3}$ neighborhood ( $D_{\text {Euclidean }}$ ), and divided by the corresponding Euclidean distances of isotropic voxels of length 1 ( $\left.D_{\text {Euclidean }_{\text {iso }}}\right)$ to compute the compensation factor $(\lambda$, Eq. 1$)$ for each position in the $3^{3}$ or $5^{3}$ neighborhood. The values of $D_{\text {opt }}$ are multiplied by $\lambda$ as in Eq. 2 to determine the new estimator values ( $\left.D_{\text {adjusted }}\right)$. Fig. 2 tabulates the published estimator values and illustrates how these equations are applied.

$$
\begin{aligned}
\lambda & =\frac{D_{\text {Euclidean }}}{D_{\text {Euclidean }_{\text {iso }}}} \\
D_{\text {adjusted }} & =\lambda \cdot D_{\mathrm{opt}}
\end{aligned}
$$




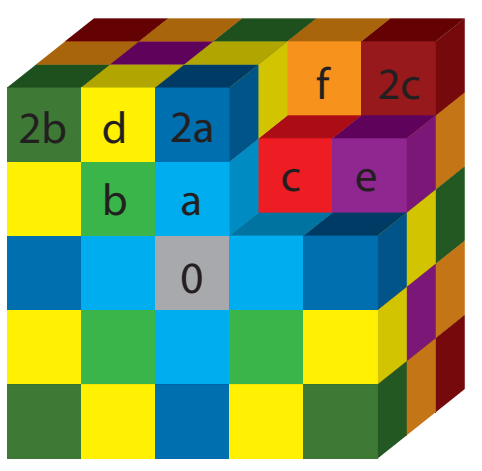

$\begin{array}{ccc}\text { Euclidean } & \text { Verwer }_{5} & \text { Verwer }_{3} \\ 1.0000 & 0.9556 & 0.8940 \\ 1.4142 & 1.3956 & 1.3409 \\ 1.7321 & 1.7257 & 1.5879 \\ 2.2361 & 2.1830 & \\ 2.4495 & 2.3885 & \\ 3.0000 & 2.9540 & \end{array}$
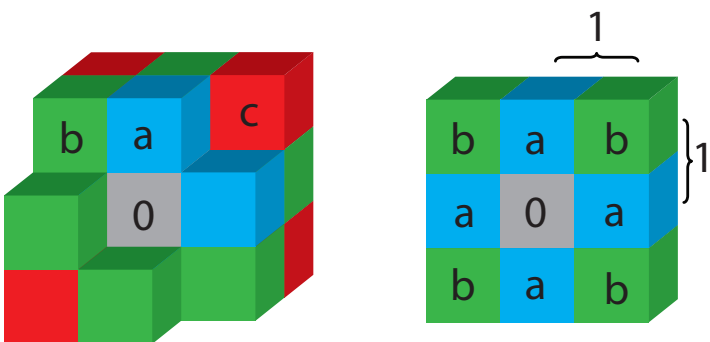

Kiryati $_{3}$

0.9016

1.289

1.615

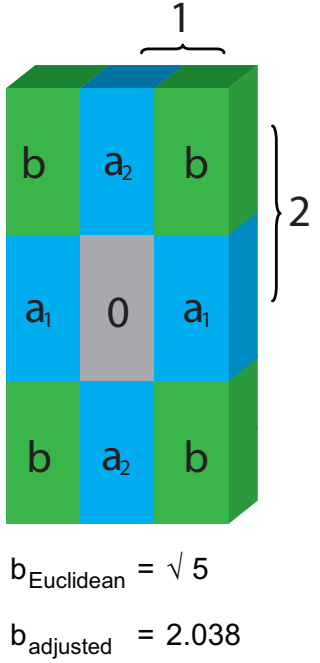

Fig. 2. Distance estimators and anisotropy adjustment. At the left side the $3^{3}$ and $5^{3}$ neighborhood is shown with the corresponding values of the published distance estimators which are valid for isotropic data sets. At the right, an adjustment, that has to be applied to each of the neighboring voxels in the $3^{3}$ neighborhoods of the center voxel is illustrated. This adjustment factor $\lambda$ equation (1) for voxel $b$ is computed $a s \lambda_{b}=b_{\text {Euclidean }} / b_{\text {Euclidean }_{\text {iso }}}=$ $\sqrt{5} / \sqrt{2}=1.5811$. Subsequently the adjusted value can be determined: $b_{\text {adjusted }}=\lambda_{b} \cdot b_{\text {Kiryati }_{3}}=1.5811 \cdot 1.289=$ 2.038 .

\section{PERFORMANCE OF THE DIFFERENT DISTANCE ESTIMATORS}

Fig. 3 shows the distances observed when using the Euclidean $_{3}$, without any optimization. The distances were continuously overestimated and a RMS error of over $9 \%$ for the isotropic model and up to $24 \%$ for the model with the highest degree of anisotropy was found. Only in the case of a straight line, at an angle of $90^{\circ}$, the estimated distance was correct.

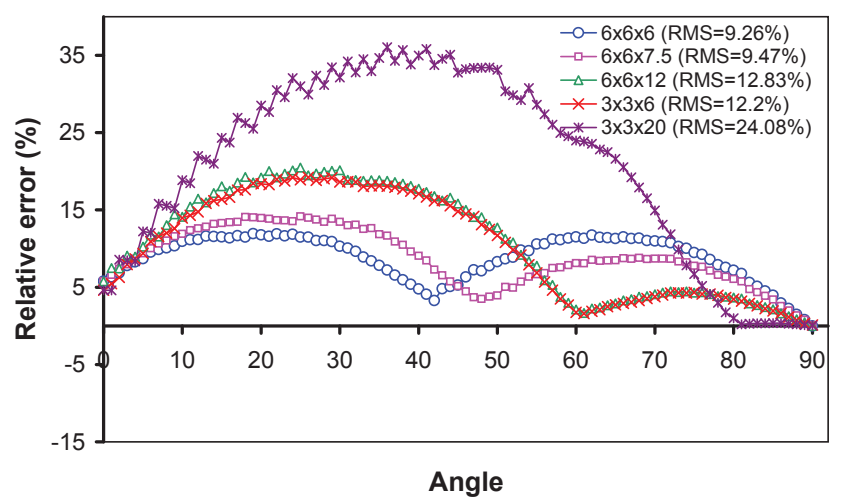

Fig. 3. Euclidean 3 estimator. For each tested resolution of the reference model the relative error compared to the expected distances is computed for the Euclidean 3 estimator.
Fig. 4 shows the distances observed when using the adjusted Kiryati $_{3}$ estimator. For the cubic model the errors were nicely centered around $0 \%$. In a model with slightly anisotropic voxels the errors were somewhat increased. In the test models with voxel sizes twice as large in the $z$ direction as in the $x y$ direction the errors increased significantly. Especially for distances measured at smaller angles, the estimator gave a large overestimation of the actual length. Comparison of the results of the two models with a factor 2 difference in $x y z$ resolution shows that a higher resolution (smaller voxels) gives slightly lower errors. A more pronounced difference is seen in the noise of the errors; the largest voxels result in a saw-tooth pattern of the error graph. In the model in which the $z$ size of the voxels was about 7 times larger than the $x y$ size much higher errors than in all previous tests were observed. The errors are especially high for angles between $20^{\circ}$ and $60^{\circ}$.

As shown in Fig. 5, the pattern of errors observed for the adjusted Verwer $_{3}$ estimator was very similar to those of Kiryati $_{3}$, although the RMS errors found were all slightly higher. 


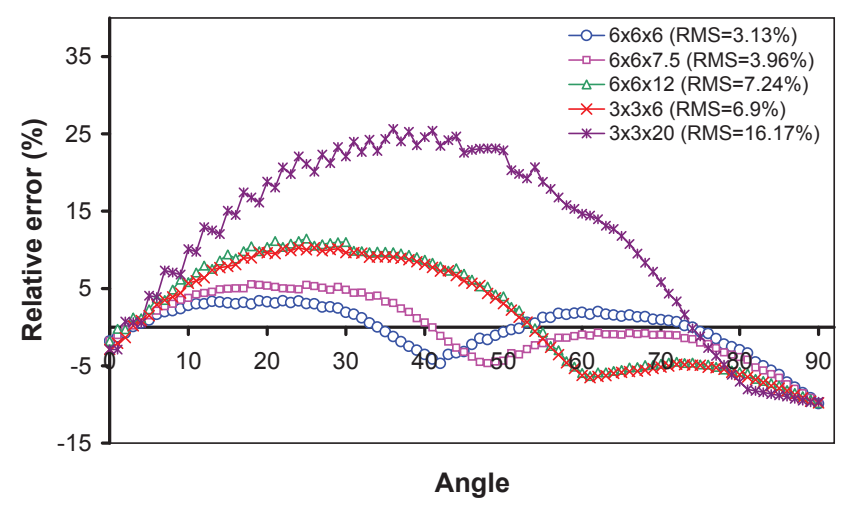

Fig. 4. Kiryati iz estimator. For each tested resolution of the reference model the relative error compared to the expected distances is computed for the adjusted Kiryati ${ }_{3}$ estimator.

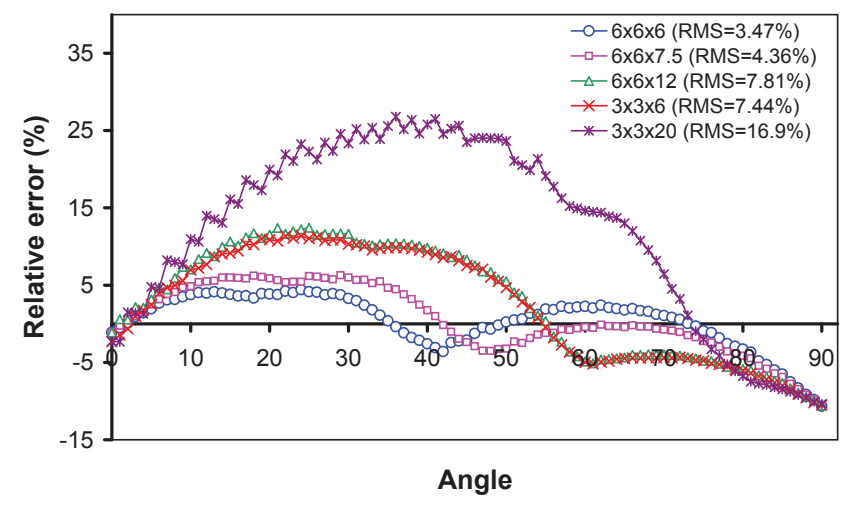

Fig. 5. Verwer 3 estimator. For each tested resolution of the reference model the relative error compared to the expected distances is computed for the adjusted Verwer $_{3}$ estimator.

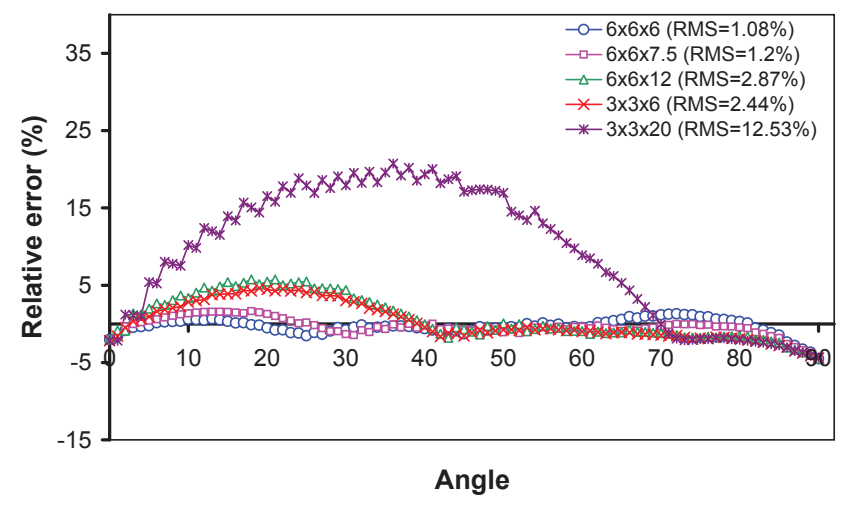

Fig. 6. Verwer 5 estimator. For each tested resolution of the reference model the relative error compared to the expected distances is computed for the adjusted Verwer 5 estimator
The $5^{3}$ neighborhood estimator of Verwer has much lower errors in all tested models which is immediately clear from the comparison of the test results (Fig. 6) to those found with the $3^{3}$ neighborhood estimators (Figs. 4 and 5). There was only a minor effect of slightly increasing the level of anisotropy (an 0.12 percent point higher RMS error between $6 \times 6 \times 6 \mu \mathrm{m}$ and $6 \times 6 \times 7.5 \mu \mathrm{m}$ voxels). Even with voxel sizes twice as large in the $z$ direction as in the $x y$ direction, the errors of the Verwer 5 estimator stay below the error levels of both $3^{3}$ neighborhood estimators in the case of isotropic voxels. Again, when comparing the results of the test with a factor 2 difference in $x y z$ resolution it is found that a higher resolution gives slightly lower errors, and the same saw-tooth pattern of the error graph is seen in the lower resolution. In the last model the $z$ size of the voxels was about 7 times larger than the $x y$ size and again much higher errors than in the previous tests with this estimator were seen. However, the Verwer $_{5}$ estimator still performed somewhat better than the other estimators in this model.

RMS errors and mean errors of the 3 adjusted estimators are summarized in Fig. 7. Up to anisotropy of 2 times all estimators are almost unbiased but the Verwer $_{5}$ estimator was by far the most precise showing the smallest RMS errors. All estimators fail when they are applied to models with levels of anisotropy of 7 times.

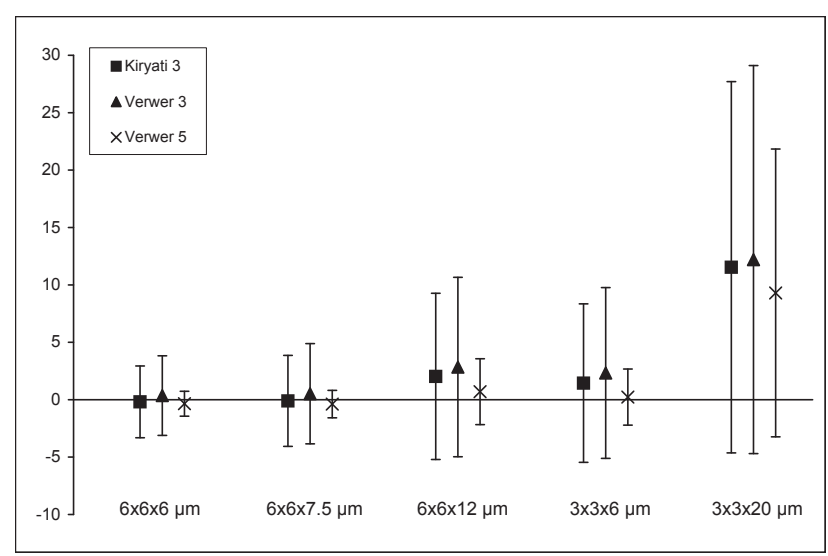

Fig. 7. The mean errors (bias) \pm the RMS error for every adjusted estimator and test.

\section{IMPLEMENTATION AND APPLICATION OF THE DISTANCE ESTIMATOR}

The results prompted us to implement the Verwer $_{5}$ estimator in an easy to use application dubbed Distance3D. We used Matlab (The Mathworks Inc.) to build the graphical user interface (Fig. 8). We optimized the program to handle data sets from 
Amira (Visage Imaging), a program often used for 3D reconstruction of biological tissue. 3D volume data from Amira, exported as multi-page (3D) tiff files, can be loaded into Distance3D whereby the voxel size information is preserved. The same holds for the surface data which is used for visualization. The latter can be exported as '*.m'-file, or when preserving color information as VRML-file. By navigating through the volume data data the way-points can be placed through which the shortest path will be determined. The waypoints will be processed in the same order as they were placed.

The program described above is used to measure the length of the BrdU-positive zone within a timed series of developing chicken hearts (Fig. 9). The growth of the heart tube due to addition of cells from a dividing precursor pool is reflected by the length of this zone. From the measurements of these lengths at different time points it can be inferred that the chicken heart tube lengthens at a rate of $70 \mu \mathrm{m} / \mathrm{h}$ (Fig. 9) as shown by van den Berg et al. (2009).

\section{DISCUSSION}

Comparison of published distance estimators showed that our approach gave slightly higher RMS errors than Verwer and Kiryati found in their measurements when measuring in a cubic grid of voxels; the RMS errors were 1.14 and 0.25 percent point higher than the $2.33 \%$ and $2.88 \%$ originally published by Verwer and Kiryati, respectively (Verwer, 1991; Kiryati and Szekely, 1993). The RMS error of $0.78 \%$ that Verwer determined for the $5^{3}$ estimator was only 0.3 percent point lower than what we found in our measurement with the Verwer 5 estimator in isotropic voxels (Verwer, 1991). Our test included a line exactly in the $z$-direction, which in a distance estimator has the maximum possible error, and also lines close to this angle. This and the limited number of test directions that we used might have lead to an overrepresentation of paths with relatively large errors in our test set. Additionally we opted for a representative test and therefor used a test set with limited lengths which might be another reason for these increased RMS errors; the limited number of voxels included in each path could have lead to extra error due to rounding-off of the pixel locations.

The reason that the Kiryati 3 estimator performs slightly better than the Verwer 3 estimator is probably caused by the basic principles of these estimators. The estimator of Kiryati is optimized for curved paths, whereas the estimators of Verwer are optimized for straight lines. The lines in the models that we have tested are almost all curved, which favors the Kiryati $_{3}$ estimator.

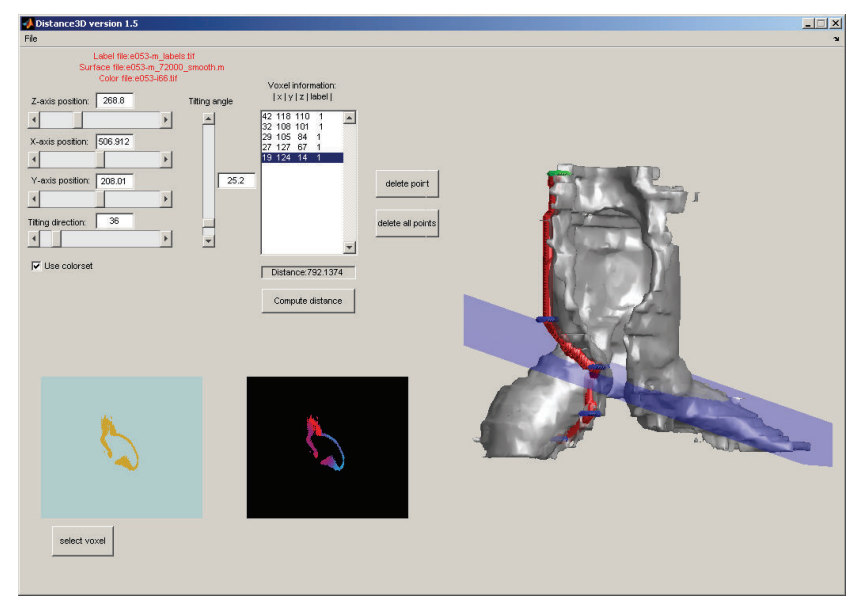

Fig. 8. Screenshot of the user interface of Distance $3 D$. The user can navigate through the volume data by handling the sliders (top left). When the desired position is found, way-points have to be placed on the cross section (bottom left). When at least two waypoints (a start and end position) are placed the shortest path can be determined and the distance computed. When more way-points are placed, the shortest path follows the way-points in the order in which they are placed.
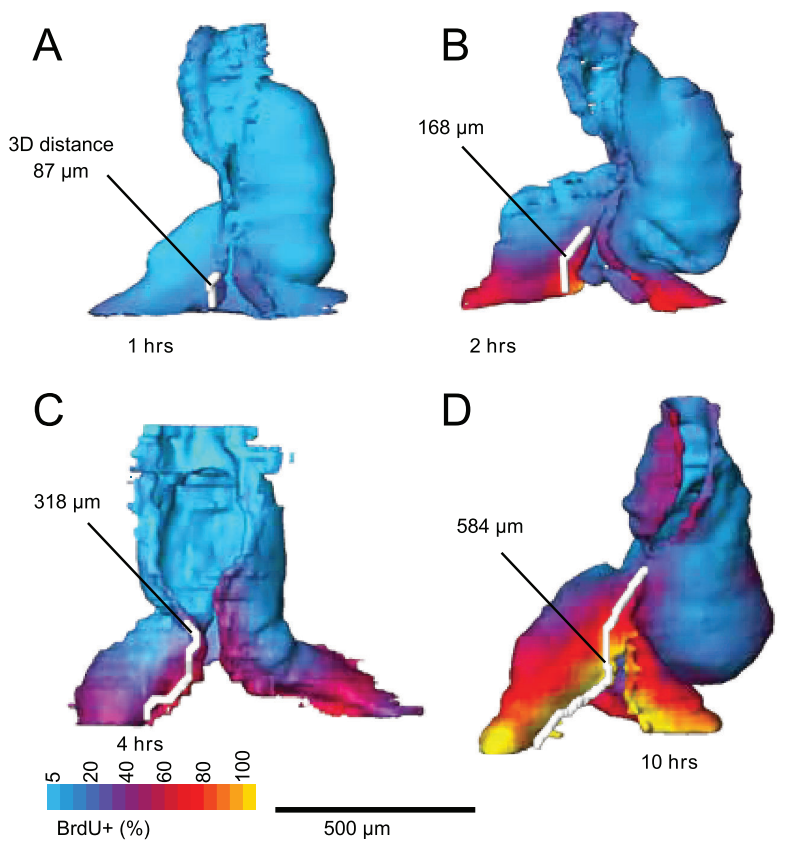

Fig. 9. Distance measurements of the length of the BrdU-positive zone in the developing embryonic chicken heart. BrdU-negative tissue is shown in blue. A lengthening of approximately $70 \mu \mathrm{m} / \mathrm{h}$ can be derived from this measurement. (figure adapted from van den Berg et al., 2009) 
An elongation of $25 \%$ in the $z$-direction would be a reasonable value for reconstructions of microscopic images resampled to near-cubic voxels. Compared to cubic voxels the RMS errors increase slightly, although this is hardly noticeable in the Verwer 5 estimator with an increase of only 0.12 percent point. The estimators in the $3^{3}$ neighborhood suffer more from this low level of anisotropy with an increase of 0.8 percent point. When measuring within biological tissue such error rates are still within an acceptable range and because the mean error stays close to zero (Fig. 7) the estimators can still be considered unbiased up to this $25 \%$ anisotropy.

The error rates of the $3^{3}$ neighborhood estimators double when elongating the voxels to a height twice as long as the $x y$-sizes, and their mean error is no longer close to zero (Fig. 7). On the other hand the Verwer $_{5}$ estimator still shows error rates that are slightly lower than the error rates found in cubic voxels with the $3^{3}$ neighborhood estimators (Fig. 7). The Verwer $_{5}$ estimator is still precise at this degree of anisotropy while it is unbiased with a mean error close to zero (Fig. 7).

Two observations can be made when comparing the results of the two tests with the doubled $z$-size. Firstly, the relative errors in the test model with the lower resolution $(6 \times 6 \times 12 \mu \mathrm{m})$ show saw-tooth-like variations whereas the graphs of the test model with higher resolution $(3 \times 3 \times 6 \mu \mathrm{m})$ are much smoother. The rounding-off error, which is larger when using larger voxels, easily explains this difference. More interestingly, the error graphs of the estimators in the lower resolution model are consistently slightly higher than those of the higher resolution model. For all estimators this leads to a higher mean overestimation of the actual length by approximately 0.5 percent point in the model with the larger voxel sizes (Fig. 7).

To decide whether the loss of resolution due to down-sampling data is worse than the error introduced by the anisotropy of the voxels, it is interesting to compare the tests with voxel sizes of $3 \times 3 \times 6 \mu \mathrm{m}$ and $6 \times 6 \times 6 \mu \mathrm{m}$ (Fig. 7). In the case of cubic voxels the mean error of all estimators is close to zero while in the elongated voxels only the error of the Verwer $_{5}$ estimator is unbiased. The RMS errors of all estimators are higher in the case of the elongated voxels, although the RMS error of the Verwer $_{5}$ estimator stays in an acceptable range. This leads to the conclusion that generally the effect of down-sampling to larger isotropic voxels, and the loss in associated spatial resolution, is not as bad as the effect of anisotropy.

The performance of all estimators in the test model with strongly elongated voxels is poor. A large overestimation of the distance is observed especially for the lines with an angle between approximately $20^{\circ}$ to $60^{\circ}$. Using these elongated voxels would result in a strongly biased estimation with mean errors around $10 \%$ (Fig. 7). Reasonable error rates are only observed for the Verwer 5 estimator above an angle of $65^{\circ}$. This shows that measuring in the direction of the voxel elongation gives lower errors, although this is associated with a bias to underestimation of the actual path. It is therefore not recommended, to estimate distances in such strongly elongated voxels . However, sometimes it is required to do measurements through very thin tissues, where down-sampling might lead to the loss of connectivity. Without this connectivity the shortest path cannot be computed. In these cases one could consider to use the highly anisotropic data set and restrict the measurements to the $z$-direction. However, downs-ampling to voxels that are up to twice as high in the $z$ direction as in the $x y$ direction should always be attempted because at that level of anisotropy the adjusted $5^{3}$ neighborhood estimator of Verwer, showed errors that are acceptable for all directions.

The tests we performed using a model representing a simplified early heart tube resulted in length measurements over curves. Similar tests have to be performed to test the performance in structures with other geometric characteristics. We expect that the Verwer $_{5}$ estimator will perform even better in structures with a more solid composition because it was originally optimized for such straight lines.

The approach we used to adapt the published optimized values for anisotropic voxels does not by definition result in the best values for every degree of anisotropy. However, the results are sufficiently precise for biological applications as long as voxel sizes in the $z$ direction are not longer as twice the $x y$ size.

Using Farey sets, Fouard and Malandain (2005) computed optimal integer approximations for anisotropic voxels. These estimators were found by minimizing the maximum error, without taking the RMS error and mean error into account. Only when the application requires a higher precision than usually required in biology, this computationally expensive approach would be worth considering.

\section{CONCLUSION}

Existing length estimators can be very simply adjusted for the use within an anisotropic non-convex space using our approach.

Two conclusions can be drawn from the test results. Firstly, anisotropy of voxels has a big influence 
on the error of the estimated length of the shortest path. Secondly, the estimator that performed best in all tests was the anisotropy-adjusted $5^{3}$ neighborhood estimator of Verwer.

Because this estimator performed almost unbiased, with acceptable RMS errors, within levels of anisotropy up till voxels that are twice as high as they are wide it is recommended to re-sample data sets to such, close to cubic, voxels .

\section{FUNDING}

This work was supported by the EU seventh framework program as part of the project CHeartED [HEALTH-F2-2008-223040].

\section{REFERENCES}

Borgefors G (1984). Distance transformations in arbitrary dimensions. Comput Vision Graph 27:321-45.

Coquin D, Bolon P (1995). Discrete distance operator on rectangular grids. Pattern Recogn Lett 16:911-23.

Dijkstra EW (1959). A note on two problems in connexion with graphs. Numer Math 1:269-71.

Fouard C, Malandain G (2005). 3-d chamfer distances and norms in anisotropic grids. Image Vision Comput 23:143-58.

Howard CV, Reed MG (1998). Unbiased stereology. Threedimensional measurement in microscopy. Liverpool, UK: Bios Scientific Publishers, 1st ed.
Kiryati N, Szekely G (1993). Estimating shortest paths and minimal distances on digitized three-dimensional surfaces. Pattern Recogn 26:1623-37.

Sintorn IM, Borgefors G (2004). Weighted distance transforms for volume images digitized in elongated voxel grids. Pattern Recogn Lett 25:571-80.

Soufan AT, Ruijter JM, van den Hoff MJB, Moorman AFM (2001). Quantitative 3D reconstructions as identification tool in heart development. Image Anal Stereol 20:193-8.

Soufan AT, van den Berg G, Moerland PD, Massink MMG, van den Hoff MJB, Moorman AFM, Ruijter JM (2007). Three-dimensional measurement and visualization of morphogenesis applied to cardiac embryology. J Microsc 225:269-74.

Soufan AT, van den Berg G, Ruijter JM, de Boer PAJ, van den Hoff MJB, Moorman AFM (2006). Regionalized sequence of myocardial cell growth and proliferation characterizes early chamber formation. Circ Res 99:545-52.

van den Berg G, Abu-Issa R, de Boer BA, Hutson MR, de Boer PA, Soufan AT, Ruijter JM, Kirby ML, van den Hoff MJ, Moorman AF (2009). A caudal proliferating growth center contributes to both poles of the forming heart tube. Circ Res 104:179-88.

Verwer BJH (1991). Local distances for distance transformations in two and three dimensions. Pattern Recogn Lett 12:671-82. 